\title{
IN VIVO CONFOCAL RAMAN SPECTROSCOPY OF THE SKIN
}

\author{
P. J. Caspers*, G. W. Lucassen ${ }^{* *}$, H. A. Bruining*, G. J. Puppels ${ }^{*}$ \\ * Erasmus University Rotterdam, Department of General Surgery, Dr. Molewaterplein 40, 3015 GD \\ Rotterdam, The Netherlands \\ ** Philips Research, Personal Care Institute, Prof. Holstlaan 4, 5656 AA Eindhoven, The \\ Netherlands
}

In vivo methods that provide qualitative and quantitative information about the presence and depth distribution of molecular compounds in the skin are of great help in addressing many biological, medical and cosmetic research questions. Existing non-invasive in vivo methods provide little depth information about molecular concentrations across the epidermis [1]. In vivo confocal Raman microspectroscopy is a new method to determine the depth distribution of a range of molecular compounds in the skin [2-4]. We have established a confocal Raman microspectroscopic system dedicated to skin research. Measurements are performed directly on the skin and are fully noninvasive. The spatial resolution in the direction perpendicular to the skin surface is currently about 5 micron, which enables spatially resolved measurements within the stratum corneum (thickness about 15 micron). The depth range from which spectra can be obtained can be up to several hundred microns below the skin surface. A diagram of the Raman setup is shown in figure 1.

Skin hydration and skin moisturizers have received attention from both medicine and cosmetics. The skin itself produces a mixture of moisturizing substances in the lower part of the stratum corneum (SC). This mixture of mainly free amino acids and amino acid derivatives is referred to as natural moisturizing factor (NMF). The SC provides a highly efficient water barrier to the skin, which results in a steep water concentration gradient across the SC. Much of what is known about skin hydration, NMF and the skin barrier is based on in vitro information. We have used in vivo confocal Raman spectroscopy to determine concentration profiles across the SC of various compounds including water, and compounds of natural moisturizing factor and sweat. Figure 2 illustrates the unique possibility of in vivo confocal Raman microspectroscopy to study skin hydration noninvasively. Water concentration profiles were determined for the stratum corneum of the lower forearm. This was repeated after intense hydration for 45 minutes with a water soaked bandage. Dramatic changes in the shape of the in vivo water concentration profile is observed. Clearly the water concentration in the SC (0-15 micron) has largely increased to about $60 \%$.

\section{References:}

[1] J. Serup and G.B.E. Jemec, Non-invasive methods and the skin,CRC Press, Boca Raton,1995.

[2] P.J. Caspers et al., Biospectr. 4, (1998) S31.

[3] P.J. Caspers et al., J. Raman Spectrosc., 31 (2000) 813.

[4] P.J. Caspers et al., J. Invest. Dermatol. 116 No. 3 (2001) 434. 


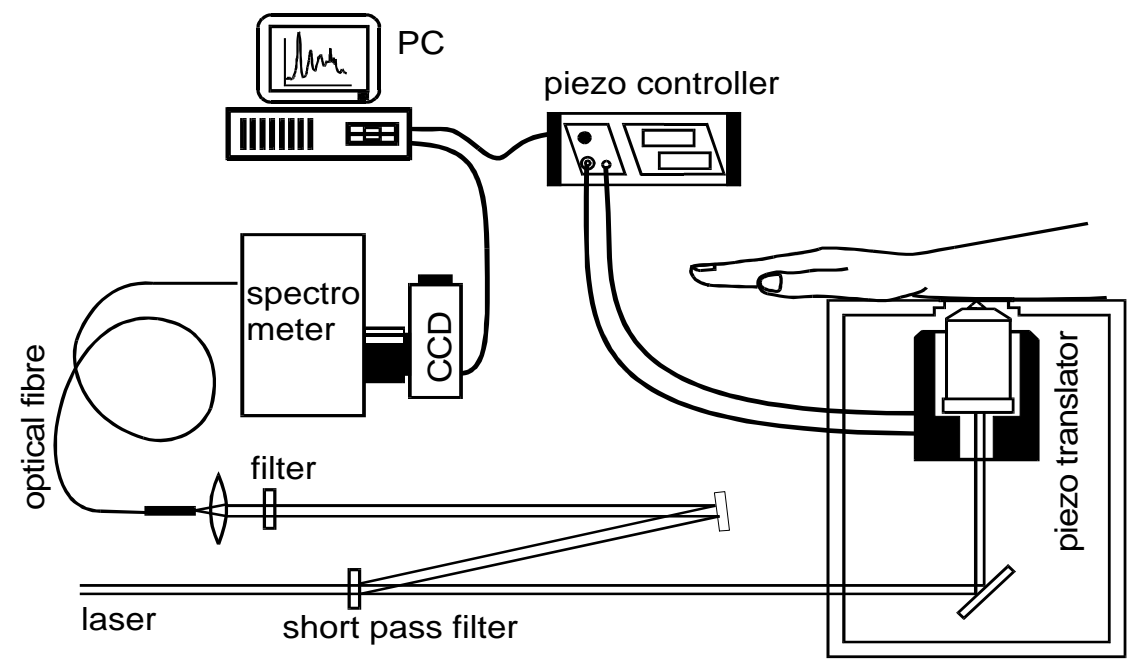

FIG. 1. Diagram of the in vivo confocal Raman microspectrometer. A laser beam is focused in the skin by a microscope objective. The Raman scattered light is reflected by a short-pass filter and coupled into an optical fiber, which is connected to the spectrometer. Vertical translation of the microscope objective changes the position - i.e. the distance to the skin surface - of the laser focus. During measurement the skin rests on a fused silica or $\mathrm{CaF}_{2}$ window, which prevents movement of the skin surface.

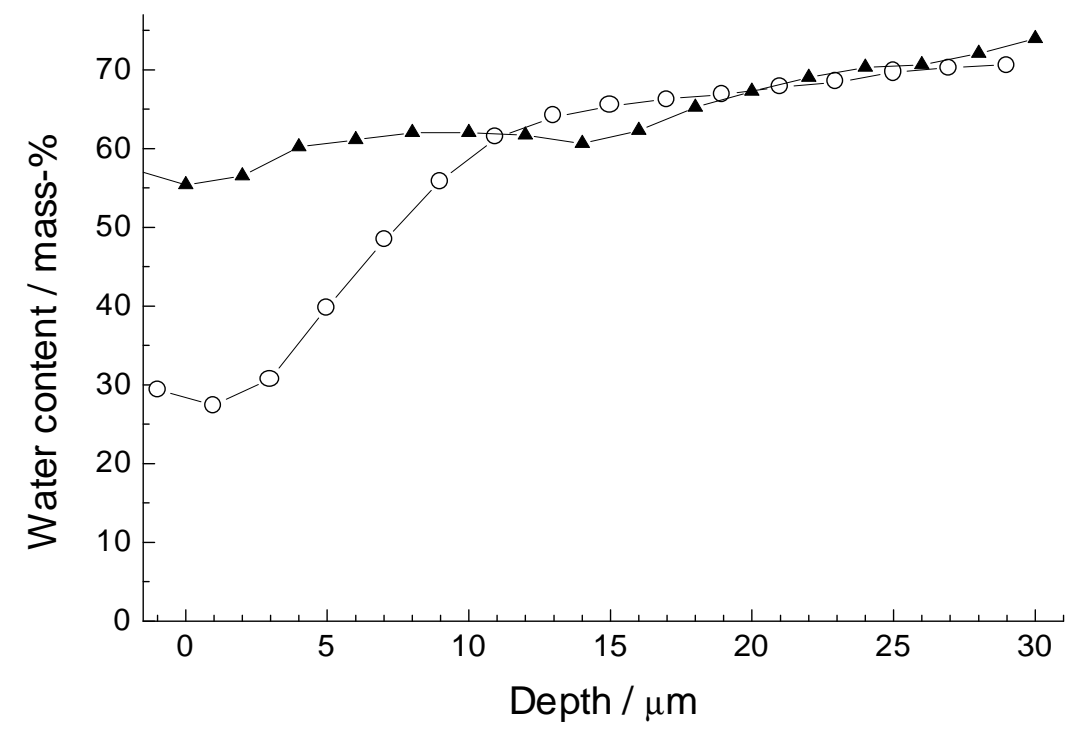

FIG. 2. In vivo water concentration profiles for the forearm before (circles) and after (triangles) hydration for 45 minutes. 